\title{
GRAVITY MODES IN ZZ CETI STARS. II. EIGENVALUES AND EIGENFUNCTIONS
}

\author{
YANQIN WU ${ }^{1}$ AND PETER GOLDREICH ${ }^{2}$ \\ Received 1998 December 3; accepted 1999 February 18
}

\begin{abstract}
We report on numerical calculations of nonadiabatic eigenvalues and eigenfunctions for $g$-modes in ZZ Ceti variables. The spectrum of overstable $l=1$ modes delineates the instability strip. Its blue edge occurs where $\omega \tau_{c} \approx 1$ for the $n=1$ mode. Here $\omega$ is radian frequency and $\tau_{c}$ is about 4 times the thermal time at the bottom of the surface convection zone. As a $\mathrm{ZZ}$ Ceti cools, its convection zone deepens, longer period modes become overstable, but the critical value of $\omega \tau_{c}$ separating overstable and damped modes rises. The latter is a consequence of enhanced radiative damping for modes that propagate immediately below the convection zone. The critical value of $\omega \tau_{c}$ is of observational significance, because modes with the smallest value of $\omega \tau_{c}$ are most observable photometrically. Maximum periods for overstable modes predicted for our cooler model envelopes are about a factor of 2 longer than the observational upper limit of $1200 \mathrm{~s}$. We assess a number of plausible resolutions for this discrepancy among which convective overshoot and nonlinear saturation look promising. The nonadiabatic eigenfunctions enable us to predict relative amplitudes and phases of photospheric variations of flux and velocity, quantities made accessible by recent observations. We also present asymptotic formula for damping rates of high-order modes, a result of consequence for future investigations of nonlinear saturation of the amplitudes of overstable modes.
\end{abstract}

Subject headings: stars: oscillations - stars: variables: other

\section{INTRODUCTION}

While passing through a narrow temperature range around $T_{\text {eff }} \sim 12,000 \mathrm{~K}$, DA (hydrogen) white dwarfs exhibit pulsations with periods between 2 and 20 minutes. These oscillations are identified with $g$-modes having radial orders $1 \leq n \leq 30$ and spherical harmonic degrees $1 \leq l \leq 2$. Mode overstability occurs when driving in the thin and fast reacting surface convection zone exceeds damping in the underlying radiative interior (Brickhill 1991; Gautschy, Ludwig, \& Freytag 1996; Goldreich \& Wu 1999a, hereafter Paper I). The flux perturbation entering the convection zone from the underlying radiative interior is in phase with the pressure perturbation. Because the convection is fast and efficient, the specific entropy perturbation is nearly depth-independent throughout the bulk of the convection zone. Consequently, the magnitude of the flux perturbation decreases outward. The phase relation between the pressure and flux perturbations then results in convective driving.

Gravity-modes have simple structures in their upper evanescent regions. This permits an analytic derivation of a stability criterion based on the quasi-adiabatic approximation for modes whose evanescent regions extend well below the base of the convection zone. Overstability is predicted provided $\omega \tau_{c}>1$, where $\tau_{c}$ is a few times the thermal timescale at the bottom of the convection zone. Applied blindly, this criterion predicts overstability in cool ZZ Cetis for modes whose periods far exceed those observed. Our

\footnotetext{
${ }^{1}$ Astronomy Unit, School of Mathematical Sciences, Queen Mary and Westfield College, Mile End Road, London E1 4NS, England, UK; Y.Wu@qmw.ac.uk.

2 Theoretical Astrophysics, California Institute of Technology, 130-33, Pasadena, CA 91125; pmg@gps.caltech.edu.
}

numerical calculations enable us to drop both the quasiadiabatic approximation and the restriction that the evanescent region extend well below the convection zone. They also allow us to incorporate the effect of turbulent viscosity as discussed in Brickhill (1990) and Goldreich \& Wu (1999b, hereafter Paper III).

This paper is the second in a series dedicated to the overstability of $g$-modes in ZZ Cetis. It is largely numerical and presents results of nonadiabatic calculations of eigenvalues and eigenfunctions. Nonadiabaticity is important for modes that have $\omega \tau_{c} \lesssim 1$, since they have $\omega \tau_{\text {th }}<1$ in their driving and damping regions. This category includes both overstable modes with $\omega \tau_{c} \approx 1$, which are easily detectable by photometric measurements, and damped modes with $\omega \tau_{c} \ll 1$, whose excitation by parametric instability acts to limit the amplitudes of overstable modes. It is imperative that our calculations accurately represent them.

The organization of this paper is as follows: In $\S 2$, we collect the equations which govern linear, nonadiabatic oscillations, establish appropriate boundary conditions, and describe the method we use to construct envelope models for ZZ Cetis. In $\S 3$ we assess the magnitude of nonadiabatic effects in the evanescent and propagating regions of $g$-modes. Numerical techniques employed to solve the eigenvalue problem are elaborated in $\S 4$. The impact of nonadiabaticity and turbulent viscosity on $g$-mode eigenvalues and eigenfunctions is described in $\S 5$. We then discuss in $\S 6$ several possibilities to reconcile the nonadiabatically calculated spectra of overstable modes with those observed. A short summary follows in $\S 7$. The Appendix is devoted to analysis of a toy model, which elucidates the asymptotic damping rates of high-order modes.

Symbols used in this paper are defined in Table 1. Their usage is consistent with that in other papers of this series. 
TABLE 1

DEFINITIONS

\begin{tabular}{|c|c|}
\hline Symbol & Meaning \\
\hline$R \ldots \ldots \ldots$ & Stellar radius \\
\hline$g \ldots \ldots \ldots . .$. & Surface gravity \\
\hline$z \ldots \ldots \ldots \ldots$ & Depth below photosphere \\
\hline$z_{b} \ldots \ldots \ldots \ldots$ & Depth at bottom of convection zone \\
\hline$z_{\omega} \ldots \ldots \ldots$ & Depth at top of mode's cavity, $z_{\omega} \sim \omega^{2} /\left(g k_{h}^{2}\right)$ \\
\hline$\omega \ldots \ldots \ldots$ & Radian mode frequency, $\omega=\omega_{r}+i \omega_{i}$ \\
\hline$n \ldots \ldots \ldots$ & Radial order of mode \\
\hline$\ell \ldots \ldots \ldots$ & Angular degree of mode \\
\hline$k_{h} \ldots \ldots \ldots$ & Horizontal wavevector, $k_{h}^{2}=\ell(\ell+1) / R^{2}$ \\
\hline$k_{z} \ldots \ldots \ldots$ & Vertical wavevector \\
\hline$\rho \ldots \ldots \ldots \ldots$ & Mass density \\
\hline$\rho_{b} \ldots \ldots \ldots \ldots$ & Mass density at $z=z_{b}$ \\
\hline$p \ldots \ldots \ldots . .$. & Pressure \\
\hline$T \ldots \ldots \ldots$ & Temperature \\
\hline$s \ldots \ldots \ldots \ldots$ & Specific entropy in units of $k_{B} / m_{p}$ \\
\hline$F \ldots \ldots \ldots$ & Energy flux \\
\hline$N^{2} \ldots \ldots \ldots$ & Brunt-Väisälä frequency \\
\hline$c_{s} \ldots \ldots \ldots \ldots$ & Adiabatic sound speed, $c_{s}^{2}=(\partial p / \partial \rho)_{s}=\Gamma_{1} p / \rho$ \\
\hline$\rho_{s} \ldots \ldots \ldots$ & $\rho_{s} \equiv(\partial \ln \rho / \partial s)_{p}$ \\
\hline$c_{p} \ldots \ldots \ldots$ & Dimensionless heat capacity, $c_{p} \equiv(\partial s / \partial \ln T)_{p}$ \\
\hline$\kappa_{T}, \kappa_{\rho} \ldots \ldots$ & Opacity derivatives, $\kappa_{T} \equiv(\partial \ln \kappa / \partial \ln T)_{\rho}, \kappa_{\rho} \equiv(\partial \ln \kappa / \partial \ln \rho)_{T}$ \\
\hline$\nabla \ldots \ldots \ldots$ & Temperature gradient, $\nabla \equiv(d \ln T / d \ln p)$ \\
\hline$\nabla_{\mathrm{ad}} \ldots \ldots \ldots$ & Adiabatic temperature gradient, $\nabla_{\mathrm{ad}} \equiv(\partial \ln T / \partial \ln p)_{s}$ \\
\hline$f \ldots \ldots \ldots \ldots$ & Coefficient measuring convective inefficiency \\
\hline$v_{\mathrm{cv}} \ldots \ldots \ldots$ & Convective velocity, $v_{\mathrm{cv}} \sim(F / \rho)^{1 / 3}$ \\
\hline$t_{\mathrm{cv}} \cdots \cdots \cdots$ & Response time for convection, $t_{\mathrm{cv}} \sim z / v_{\mathrm{cv}}$ \\
\hline$B, C \ldots \ldots$ & Dimensionless constants relating $\delta s$ and $\delta F / F$ at photosphere and across superadiabatic layer; each $\approx 8$ \\
\hline$\tau_{\text {th }} \ldots \ldots \ldots$ & Thermal time at depth $z ; t_{\mathrm{cv}} / \tau_{\mathrm{th}} \sim\left(v_{\mathrm{cv}} / c_{s}\right)^{2}$ in the convection zone \\
\hline$\tau_{c} \ldots \ldots \ldots \ldots$ & Time constant of low pass filter for convection zone, $\tau_{c} \approx(B+C) \tau_{\mathrm{th}}\left(z_{b}\right) / 5$ \\
\hline$\tau_{\omega} \ldots \ldots \ldots$ & Thermal time at $z=z_{\omega}$ \\
\hline$\delta \ldots \ldots \ldots \ldots$ & Denotes Lagrangian perturbation \\
\hline$\xi_{h} \ldots \ldots \ldots .$. & Horizontal component of displacement vector \\
\hline$\xi_{z} \ldots \ldots \ldots$ & Vertical component of displacement vector \\
\hline$v_{h} \ldots \ldots \ldots \ldots$ & Horizontal component of velocity vector \\
\hline
\end{tabular}

\section{EQUATIONS AND BOUNDARY CONDITIONS}

\subsection{Equations in the Radiative Region}

The linearized equations describing nonadiabatic pulsations read

$$
\begin{aligned}
\frac{\delta \rho}{\rho} & =-i k_{h} \xi_{h}-\frac{d \xi_{z}}{d z}, \\
\frac{\omega^{2}}{g} \xi_{h} & =i k_{h}\left(\frac{p}{g \rho} \frac{\delta p}{p}-\xi_{z}\right), \\
\frac{\omega^{2}}{g} \xi_{z} & =\frac{p}{\rho g} \frac{d}{d z}\left(\frac{\delta p}{p}\right)-\frac{d \xi_{z}}{d z}+\frac{\delta p}{p}-\frac{\delta \rho}{\rho}, \\
\delta s= & \frac{i F m_{p}}{\omega \rho k_{B} T} \frac{d}{d z}\left(\frac{\delta F}{F}\right), \\
\frac{\delta F}{F}= & \left(4-\kappa_{T}\right) \frac{\delta T}{T}-\left(1+\kappa_{\rho}\right) \frac{\delta \rho}{\rho} \\
& -\frac{d \xi_{z}}{d z}+\frac{p}{\rho g \nabla} \frac{d}{d z}\left(\frac{\delta T}{T}\right),
\end{aligned}
$$

for plane-parallel geometry with the Cowling approximation and an assumed time-dependence of $e^{-i \omega t}$. Equations (1)-(4) express the conservation of mass, momentum, and energy. Equation (5) describes radiative transfer in the diffusion approximation. Closure of this system of equations requires constitutive relations for the equation of state and the opacity. Setting $\delta s=0$ reduces the above equations to the adiabatic ones studied in Paper I.

We choose $\delta p$ and $\delta s$ as our independent thermodynamic variables and set

$$
\begin{gathered}
\frac{\delta \rho}{\rho}=\frac{1}{\Gamma_{1}} \frac{\delta p}{p}+\rho_{s} \delta s, \\
\frac{\delta T}{T}=\nabla_{\mathrm{ad}} \frac{\delta p}{p}+\frac{\delta s}{c_{p}} .
\end{gathered}
$$

Then the linear perturbation equations may be written as four first-order differential equations for the four dependent variables $\delta p / p, d(\delta p / p) / d \ln p, \delta F / F$, and $\delta s$;

$$
\begin{aligned}
\frac{d}{d \ln p}\left(\frac{\delta p}{p}\right)= & X \\
\frac{d X}{d \ln p}= & -\left(\frac{p}{g \rho}\right)^{2}\left[k_{h}^{2}\left(\frac{N^{2}}{\omega^{2}}-1\right)+\left(\frac{\omega}{c_{s}}\right)^{2}\right]\left(\frac{\delta p}{p}\right) \\
& -X+\left(\frac{p}{g \rho}\right) \frac{\left(g k_{h}\right)^{2}-\omega^{4}}{g \omega^{2}} \rho_{s} \delta s \\
\frac{d}{d \ln p}\left(\frac{\delta F}{F}\right)= & \frac{-i \omega k_{B} T p}{g m_{p} F} \delta s
\end{aligned}
$$




$$
\begin{aligned}
\frac{1}{c_{p} \nabla} \frac{d \delta s}{d \ln p}= & -\left[\left(4-\kappa_{T}\right) \nabla_{\mathrm{ad}}-\frac{\kappa_{\rho}}{\Gamma_{1}}-\left(1-\frac{\omega^{2} p}{g^{2} \rho}\right)\right. \\
& \left.\times \frac{\left(g k_{h}\right)^{2}}{\left(g k_{h}\right)^{2}-\omega^{4}}+\frac{1}{\nabla} \frac{d \nabla_{\mathrm{ad}}}{d \ln p}\right]\left(\frac{\delta p}{p}\right) \\
& -\left[\frac{\nabla_{\mathrm{ad}}}{\nabla}-\frac{\left(g k_{h}\right)^{2}}{\left(g k_{h}\right)^{2}-\omega^{4}}\right] X+\left(\frac{\delta F}{F}\right) \\
& -\left[\frac{\left(4-\kappa_{T}\right)}{c_{p}}-\kappa_{\rho} \rho_{s}+\frac{1}{\nabla} \frac{d}{d \ln p}\left(\frac{1}{c_{p}}\right)\right] \delta s .
\end{aligned}
$$

The perturbation equations are to be solved as an eigenvalue problem. Since equation (10) includes a factor $i$, all four dependent variables and the eigenfrequency are complex. We write each complex variable in the form

$$
Q=\left(Q_{r}+i Q_{i}\right) \exp ^{-i \omega t} .
$$

The physical perturbation is given by $\operatorname{Re}(Q)=$ $e^{\omega_{i} t}\left(Q_{r} \cos \omega_{r} t+Q_{i} \sin \omega_{r} t\right)$.

\subsection{Equations in the Convection Zone}

Inside the convection zone, $g$-mode perturbations are constrained by the rapid response of convection to the instantaneous pulsational state (Brickhill 1990, 1991; Papers I and III). Rapid momentum diffusion enforces

$$
|X|=\left|\frac{d}{d \ln p}\left(\frac{\delta p}{p}\right)\right| \ll \frac{z}{z_{\omega}}\left|\frac{\delta p}{p}\right|,
$$

and fast entropy mixing ensures that

$$
\left|\frac{d \delta s}{d \ln p}\right| \ll \frac{z}{z_{\omega}}|\delta s|,
$$

except in the thin superadiabatic layer. The entropy gradient in the superadiabatic layer is nonnegligible and increases with increasing convective flux. The total, convective plus radiative, flux perturbation follows from equation (10), whereas equation (11) determines the radiative flux perturbation in terms of $\delta p$ and $\delta s$.

\subsection{Boundary Conditions}

Solving four linear, homogeneous, first-order, ordinary differential equations to obtain eigenvalues and eigenfunctions requires a total of five boundary conditions. Four of these express physical constraints imposed by the environment outside the domain in which the differential equations are to be integrated. The fifth merely sets the magnitude scale and phase for the eigenfunctions.

Approximations described in $\S 2.2$ enable us to lower the outer boundary from the photosphere to the top of the radiative interior at $z_{b}$. The three boundary conditions applied there read as follows:

$$
\begin{aligned}
\left(\frac{\delta p}{p}\right) & =\text { const } \\
X & =\frac{-k_{h}^{2}}{\omega^{2} p_{b}} \int_{0}^{p_{b}} d p \frac{p \rho_{s}}{\rho}\left[\frac{d s}{d \ln p}\left(\frac{\delta p}{p}\right)-\delta s\right], \\
\delta s & =\frac{(B+C)}{1-i \omega \tau_{c}}\left(\frac{\delta F}{F}\right) .
\end{aligned}
$$

The constant on the right-hand side of equation (15) sets the scale and phase of the eigenfunction. Equation (16) follows from the near vanishing of $X$ in the convection zone, as expressed by equation (13), together with equations (20) and (21) of Paper III, which account for the jump in $X$ across the convective-radiative boundary. Equation (17) relates the entropy perturbation in the main part of the convection zone to the flux perturbation that enters from below. The constants $B$ and $C$ are defined in Paper I. The former relates the photospheric entropy perturbation to the fractional photospheric flux perturbation, whereas the latter relates the fractional perturbation of the entropy jump across the superadiabatic layer to the fractional photospheric flux perturbation. For ZZ Cetis, $B \approx C \approx 8$.

It is advantageous to raise the bottom boundary from the center of the star to a depth, $z=z_{\text {deep }}$, where the quasiadiabatic approximation is valid and the plane-parallel approximation still applies. This step alters the spectrum of eigenvalues $\omega$. However, a simple procedure to be described later allows us to recover values for $\omega_{i}$ appropriate to a complete stellar model. We generally take $z=z_{\text {deep }}$ to be the level at which $p=10^{16}$ dynes $\mathrm{cm}^{-2}$. Since $\tau_{\text {th }} \approx 10^{10} \mathrm{~s}$ at $z_{\text {deep }}$, the quasi-adiabatic approximation is valid there for all $g$-modes of interest to our investigation. And in DA white dwarf models provided to us by Bradley (1996), the region above $z_{\text {deep }}$ extends over the outer $2 \%$ of the stellar radius and includes about $10^{-6}$ of the stellar mass. The two boundary conditions imposed at $z=z_{\text {deep }}$ are

$$
\begin{aligned}
X+\left(1-\frac{k_{h}^{2} p}{\omega^{2} \rho}\right) \frac{\delta p}{p} & =0, \\
\left(\frac{\delta F}{F}\right)+M_{1}\left(\frac{\delta p}{p}\right)+M_{2} X & =0 .
\end{aligned}
$$

The mechanical boundary condition given by equation (18) states that $\xi_{z}=0$ at $z=z_{\text {deep }}$. It follows from equations (2) and (3). Thus our model is bounded from below by a rigid wall. Equation (19) is our thermal boundary condition. It is the quasi-adiabatic limit of the radiative diffusion equation (11), which defines the coefficients $M_{1}$ and $M_{2}$. Our thermal boundary condition removes the unphysical branch of the entropy perturbation that grows exponentially with depth. Dziembowski (1977) describes a more rigorous procedure for accomplishing the same result. He joins an outer nonadiabatic solution onto an inner solution comprised of a quasi-adiabatic solution for $\delta p / p$ together with an asymptotic solution for $\delta s$ that decays with depth.

Our lower boundary conditions are somewhat arbitrary. For example, we could have adopted $d \xi_{h} / d z=0$ and $\delta s=0$ as mechanical and thermal boundary conditions. These changes would not alter our conclusions about mode overstability. However, because $\delta s$ decreases rapidly with depth, taking $\delta s=0$ as the thermal boundary condition makes it more difficult for our numerical scheme to converge on eigenvalues.

\subsection{Envelope Models}

Instead of using complete white dwarf models, we work with plane-parallel hydrogen envelopes computed on fine grids. These are produced by integrating downward from the photosphere ${ }^{3}$ using the Lawrence Livermore equation

\footnotetext{
${ }^{3}$ The photosphere is taken to be where $p=2 g /(3 \kappa)$.
} 


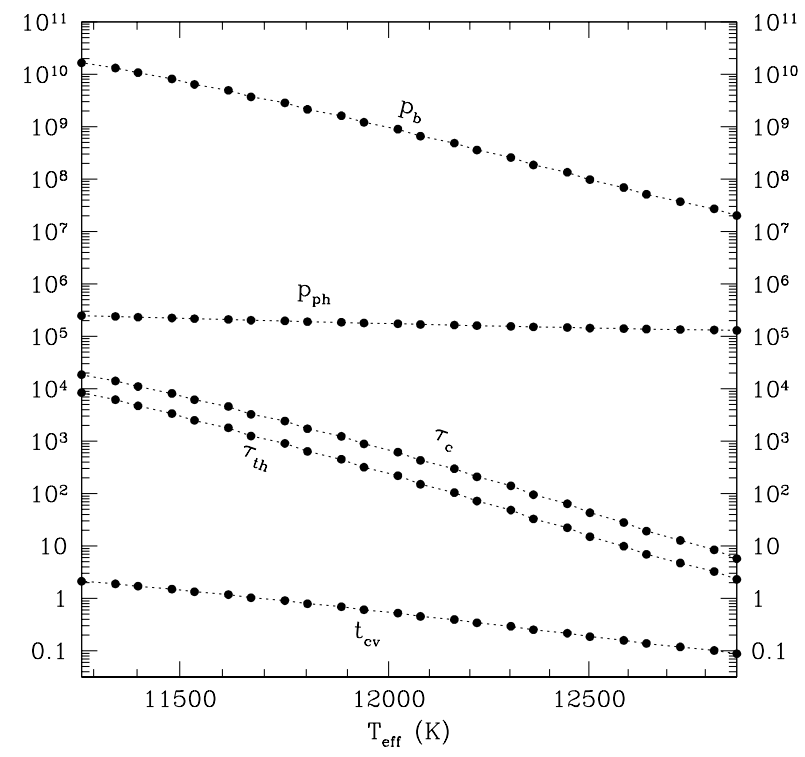

FIG. 1.-Convection zone characteristics for a sequence of hydrogen envelope models covering the temperature range where $\mathrm{ZZ}$ Cetis reside. Models have surface gravity, $g=10^{8} \mathrm{~cm} \mathrm{~s}^{-2}$, and convection parameter, $f=0.32$. Photospheric pressure, $p_{\mathrm{ph}}$, and pressure at $z_{b}, p_{b}$, are in units of dynes $\mathrm{cm}^{-2}$. Time constants $t_{\mathrm{cv}}, \tau_{\mathrm{th}}$, and $\tau_{c}$ are in units of s. Both $t_{\mathrm{cv}}$ and $\tau_{\mathrm{th}}$ are evaluated at $z_{b}$. Observationally detected $g$-modes have periods in the range $10^{2}-10^{3} \mathrm{~s}$.

of state and opacity tables (Rogers, Swenson, \& Iglesias 1996; Iglesias \& Rogers 1996). Our envelopes have $g=10^{8}$ $\mathrm{cm} \mathrm{s}^{-2}$ and cover the range $11,000 \mathrm{~K} \leq T_{\text {eff }} \leq 13,000 \mathrm{~K}$. The mass content of the convection zone in the coolest model is of order $10^{-12}$ of the stellar mass.

We model convection by invoking the mixing length Ansatz. This involves introducing a dimensionless parameter $f$ such that ${ }^{4}$

$$
\frac{d s}{d \ln p}=\frac{f}{\left(\left|\rho_{s}\right| \rho p\right)^{1 / 3}}\left(\frac{m_{p}}{k_{B}} \frac{F_{\mathrm{cv}}}{T}\right)^{2 / 3} .
$$

The parameter $f$ is of order unity and is related to the conventional mixing length ratio $\alpha$ by $f \sim \alpha^{-4 / 3}$. A smaller $f$ signifies more efficient convection and yields a thicker convection zone. The radiative flux is related to the entropy gradient by

$$
\frac{d s}{d \ln p}=\frac{3 \kappa p F_{\mathrm{rad}}}{16 \sigma T^{4} g}-\nabla_{\mathrm{ad}} .
$$

We determine $d s / d \ln p, F_{\text {cv }}$, and $F_{\text {rad }}$ from equations (20) and (21) together with the relation $F=F_{\mathrm{cv}}+F_{\mathrm{rad}}$.

The grids of our model envelopes are chosen fine enough to resolve the steep entropy profiles in the superadiabatic layer. Figure 1 displays some characteristics of the surface convection zones in envelope models produced with $f=0.32$. This value of $f$ enables us to match our model to that of Bradley's at $T_{\text {eff }}=12,420 \mathrm{~K}$. Notice that the eddy turn-over time, $t_{\mathrm{cv}}$, is of order a few seconds in even the coolest models of interest.

\section{WHERE NONADIABATICITY IS IMPORTANT}

Nonadiabaticity is quantified by how much the presence of the entropy perturbation, $\delta s$, affects $\delta p / p$ and $\delta F / F$. Our

\footnotetext{
${ }^{4}$ See eq. (23) of Paper I.
}

analysis for the radiative interior leads to separate criteria for the evanescent region and the propagating cavity.

\subsection{Nonadiabatic Effects in the Evanescent Region}

The effect of $\delta s$ on $\delta p / p$ is contained in equation (9). Noting that $\delta p / p$ varies on the scale $z_{\omega}>z$, we obtain

$$
\begin{aligned}
\frac{d}{d \ln p}\left(\frac{\delta p}{p}\right) & \sim \frac{z}{z_{\omega}}\left(\frac{\delta p}{p}\right), \\
\frac{d X}{d \ln p} & \sim \frac{z}{z_{\omega}}\left(\frac{\delta p}{p}\right), \\
\left(\frac{p}{\rho g}\right)^{2}\left[k_{h}^{2}\left(\frac{N^{2}}{\omega^{2}}-1\right)\right. & \left.+\left(\frac{\omega}{c^{2}}\right)^{2}\right]\left(\frac{\delta p}{p}\right) \\
\sim\left(\frac{k_{h} z N}{\omega}\right)^{2}\left(\frac{\delta p}{p}\right) & \sim \frac{z}{z_{\omega}}\left(\frac{\delta p}{p}\right),
\end{aligned}
$$

where $z \sim p /(g \rho)$ and $N^{2} \sim g / z$. The nonadiabatic term is of order

$$
\frac{p}{\rho g} \frac{\left(g k_{h}\right)^{2}-\omega^{4}}{g \omega^{2}} \rho_{s} \delta s \sim \frac{z}{z_{\omega}} \delta s .
$$

So the nonadiabatic correction to $\delta p / p$ is of order $\delta$.

To relate $\delta s$ to $\delta p / p$, we turn to equations (10) and (11). With appropriate scalings they yield ${ }^{5}$

$$
\begin{aligned}
\delta s & \sim \frac{1}{\omega \tau_{\mathrm{th}}} \frac{d}{d \ln p}\left(\frac{\delta F}{F}\right) \sim \frac{1}{\omega \tau_{\mathrm{th}}} \frac{\delta F}{F}, \\
\frac{\delta F}{F} & \sim \frac{\delta p}{p}+\delta s .
\end{aligned}
$$

Together, equations (23)-(24b) imply that the ratio of the nonadiabatic to adiabatic contributions to both $\delta p / p$ and $\delta F / F$ is of order $\left(\omega \tau_{\mathrm{th}}\right)^{-1}$, as is commonly cited in the literature.

\subsection{Nonadiabatic Effects in the g-Mode Cavity}

Here all perturbation quantities vary on a vertical scale $k_{z}^{-1}$, where $k_{z} z \geq 1$. Moreover, it follows from the local dispersion relation derived in Paper I that $k_{z} \sim\left(z z_{\omega}\right)^{-1 / 2}$.

Scaling the adiabatic terms in equation (9) yields

$$
\begin{aligned}
\frac{d}{d \ln p}\left(\frac{\delta p}{p}\right) & \sim\left(k_{z} z\right)\left(\frac{\delta p}{p}\right), \\
\frac{d X}{d \ln p} & \sim\left(k_{z} z\right)^{2}\left(\frac{\delta p}{p}\right), \\
\left(\frac{p}{\rho g}\right)^{2}\left[k_{h}^{2}\left(\frac{N^{2}}{\omega^{2}}-1\right)\right. & \left.+\left(\frac{\omega}{c^{2}}\right)^{2}\right]\left(\frac{\delta p}{p}\right) \\
\sim \frac{z}{z_{\omega}}\left(\frac{\delta p}{p}\right) & \sim\left(k_{z} z\right)^{2}\left(\frac{\delta p}{p}\right) .
\end{aligned}
$$

To order of magnitude, the nonadiabatic term is given by

$$
\frac{p}{\rho g} \frac{\left(g k_{h}\right)^{2}-\omega^{4}}{g \omega^{2}} \rho_{s} \delta s \sim \frac{z}{z_{\omega}} \delta s \sim\left(k_{z} z\right)^{2} \delta s .
$$

\footnotetext{
${ }^{5}$ The coefficient connecting $\delta p / p$ to $\delta F / F$ in eq. (11) varies on scale $z$, although weakly.
} 
These equations imply that the nonadiabatic correction to $\delta p / p$ is of order $\delta s$.

We scale equations (10) and (11) to relate $\delta s$ to $\delta p / p$;

$$
\begin{aligned}
\delta s & \sim \frac{\left(k_{z} z\right)}{\omega \tau_{\mathrm{th}}}\left(\frac{\delta F}{F}\right), \\
\frac{\delta F}{F} & \sim \frac{d}{d \ln p}\left(\frac{\delta p}{p}+\delta s\right) \sim\left(k_{z} z\right)\left(\frac{\delta p}{p}+\delta s\right) .
\end{aligned}
$$

Combining equations (25c)-(27b), we determine that the ratio of nonadiabatic to adiabatic contributions to $\delta p / p$ and $\delta F / F$ is of order $\left(k_{z} z\right)^{2} / \omega \tau_{\mathrm{th}}$. This is not surprising; $\tau_{\mathrm{th}} /\left(k_{z} z\right)^{2}$ is the timescale for thermal diffusion across distance $k_{z}^{-1}$. Nonadiabaticity is measured by the ratio of the mode period to this timescale.

\section{SOLUTION OF EIGENVALUE PROBLEM}

We follow a two-step procedure in solving the linear pulsation equations (8)-(11) for the four dependent variables subject to the five boundary conditions given by equations (15)-(19). The initial step consists of guessing a value for the complex eigenfrequency and then applying a relaxation method (see Press et al. 1992, chap. 17, § 3) to solve the differential equations subject to four out of the five boundary conditions. ${ }^{6}$ The step is complete when the dependent variables satisfy both the pulsation equations and the boundary conditions to $10^{-7}$ of a scaling factor provided by the corresponding adiabatic eigenfunction at individual points. Working in double precision, this is routinely achieved. The second step determines the eigenvalue by requiring the remaining boundary condition to be satisfied. Both minimization and root finding techniques work well. Normally we reserve equation (18) for our fifth boundary condition, but identical results are obtained when others are used instead.

\subsection{Eigenvalues for Stellar g-Modes}

Nonadiabatic eigenvalues of our plane-parallel hydrogen envelopes are denoted by primes, $\omega^{\prime}=\omega_{r}^{\prime}+i \omega_{i}^{\prime}$. To relate $\omega_{i}^{\prime}$ to $\omega_{i}$ of a complete DA white dwarf model, we proceed as follows: We compute adiabatic modes for a corresponding complete model and identify those whose frequencies bracket $\omega_{r}^{\prime}$. ${ }^{7}$ We determine by interpolation the noninteger number $n$ of nodes in $\delta p / p$ to assign to the adiabatic mode of the complete model, whose frequency $\omega=\omega_{r}^{\prime}$. Suppose that $n^{\prime}$ of these nodes lie above $z_{\text {deep }}$. Then

$$
\omega_{i} \approx \frac{n^{\prime}}{n} \omega_{i}^{\prime}
$$

Validation of equation (28) is provided by equation (A7) in the Appendix. Its physical justification is that, whereas energy is dissipated near the surface, it is stored throughout the mode cavity.

Our procedure for converting $\omega_{i}^{\prime}$ to $\omega_{i}$ requires nonadiabatic effects to be small well above $z_{\text {deep }}$, as is the case for all modes of interest to our investigation. However, loworder modes $(n \leq 6)$ are evanescent above $z_{\text {deep }}$. Therefore we cannot enforce the boundary condition given by equa-

\footnotetext{
${ }^{6}$ The trivial boundary condition given by eq. (15) is always included as one of this foursome.

${ }^{7}$ The adiabatic modes have the same $l$ as the mode of the plane-parallel envelope.
}

tion (18) on them. We rely on the work integral to assess the stability of these modes.

\subsection{The Work Integral}

Calculating the work integral provides an approximate method for evaluating the driving or damping rate of a mode. The work integral computes $\gamma=2 \omega_{i}$. This method is well defined and accurate when the quasi-adiabatic approximation applies. Moreover, it reveals regions of driving and damping. Since overstable $g$-modes of ZZ Cetis have highquality factors, their linear pulsations are nearly periodic. The work integral may be calculated as follows:

$$
\begin{aligned}
\gamma & =\frac{\omega_{r} R^{2}}{2 \pi} \oint d t \int_{0}^{R} d z \rho \frac{k_{B}}{m_{p}} \delta T \frac{d \delta s}{d t} \\
& =\frac{\omega_{r} R^{2}}{2} \int_{0}^{R} d z \nabla_{\mathrm{ad}} \rho \frac{k_{B}}{m_{p}} T\left[\left(\frac{\delta p}{p}\right)_{r} \delta s_{i}-\left(\frac{\delta p}{p}\right)_{i} \delta s_{r}\right]
\end{aligned}
$$

(Unno, Osaki, \& Ando 1989), where the eigenfunctions are normalized to yield mode energy equal to unity.

\section{NUMERICAL RESULTS}

\subsection{Nonadiabatic Effects on Eigenfunctions}

The effects of nonadiabaticity are illustrated by comparing nonadiabatic and adiabatic eigenfunctions for three $l=1 \mathrm{~g}$-modes of the same stellar model. The model is characterized by $T_{\text {eff }}=12,000 \mathrm{~K}, g=10^{8} \mathrm{~cm} \mathrm{~s}^{-2}$, and $f=0.32$, which together imply $\tau_{\text {th }} \approx 200 \mathrm{~s}$ at $z=z_{b}$. The modes have periods of 430, 1500, and 2400 s. Quasi-adiabatic calculations predict overstability for each of these modes.

Radiative diffusion acts to soften sharp temperature gradient perturbations. Its importance increases with mode period. These characteristics are illustrated in Figures 2-4. ${ }^{8}$ The $430 \mathrm{~s}$ mode, with $\omega \tau_{\mathrm{th}}=2.8$ at $z=z_{b}$, is quite adiabatic in the radiative interior. Radiative diffusion is more pronounced in the evanescent region of the $1500 \mathrm{~s}$ mode, which has $\omega \tau_{\mathrm{th}}=0.8$ at $z=z_{b}$. The nonadiabatic eigenfunction of the $2400 \mathrm{~s}$ mode, for which $\omega \tau_{\text {th }}=0.5$ at $z=z_{b}$, deviates significantly from its adiabatic counterpart in both the evanescent and propagating regions. The phase shift between $\delta F / F$ and $\delta p / p$ at $z_{b}$ is so large that the convection zone contributes to mode damping. This mode is stable.

Contributions of different regions to mode driving and damping are illustrated by plots of the differential work, defined by

$$
\frac{d W}{d \log p}=\frac{\omega_{r} R^{2}}{2} \nabla_{\mathrm{ad}} \frac{p k_{B} T}{g m_{p}}\left[\left(\frac{\delta p}{p}\right)_{r} \delta s_{i}-\left(\frac{\delta p}{p}\right)_{i} \delta s_{r}\right] .
$$

The mode driving at the top of the radiative interior seen in Figures 2 and 3 is a nonadiabatic effect; quasi-adiabatic calculations predict mode damping in this region. The outward decrease of the radiative flux perturbation is the immediate cause of the nonadiabatic driving. This has nothing to do with the kappa effect. It arises because trapping of the perturbed flux in the convection zone causes the temperature perturbation at the radiative convective boundary to exceed the adiabatic value. Differential work inside the convection zone is computed by setting $\delta s=\delta s_{b}$.

\footnotetext{
${ }^{8}$ We normalize $\delta p / p$ at $z=z_{\text {deep }}$ by setting it equal to its adiabatic counterpart. Thus the imaginary component of $\delta F / F$ is due to nonadiabaticity.
} 


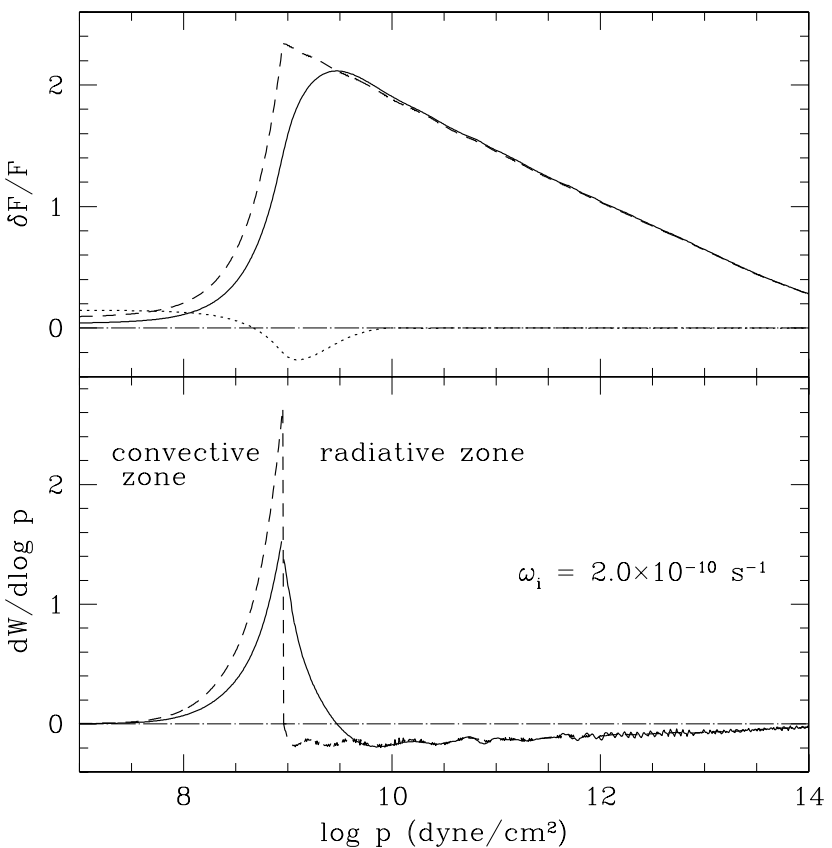

FIG. 2.-Comparisons of eigenfunctions calculated with and without the inclusion of radiative diffusion. The upper panel shows the fractional Lagrangian flux perturbation, $\delta F / F$, and the lower panel shows the differential work, both in arbitrary units and plotted against $\log p$. Results from quasi-adiabatic calculations are depicted by dashed lines, whereas those from nonadiabatic calculations are given by solid lines for real parts and dotted lines for imaginary parts. We set the nonadiabatic $\delta F / F$ to have the same phase and amplitude as the quasi-adiabatic one in the deep adiabatic interior. The stellar model has $T_{\text {eff }}=12,000 \mathrm{~K}$, which with $g=10^{8} \mathrm{~cm} \mathrm{~s}^{-2}$ and $f=0.32$ yields $\tau_{\text {th }}=200 \mathrm{~s}$ at $z_{b}$. The mode shown here has $l=1$ and $P=430 \mathrm{~s}$. It is fairly adiabatic in the radiative region since $\omega \tau_{\text {th }}=2.8$ at $z_{b}$. Nevertheless, radiative diffusion noticeably reduces convective driving and gives rise to a small region of radiative driving immediately below the convection zone.

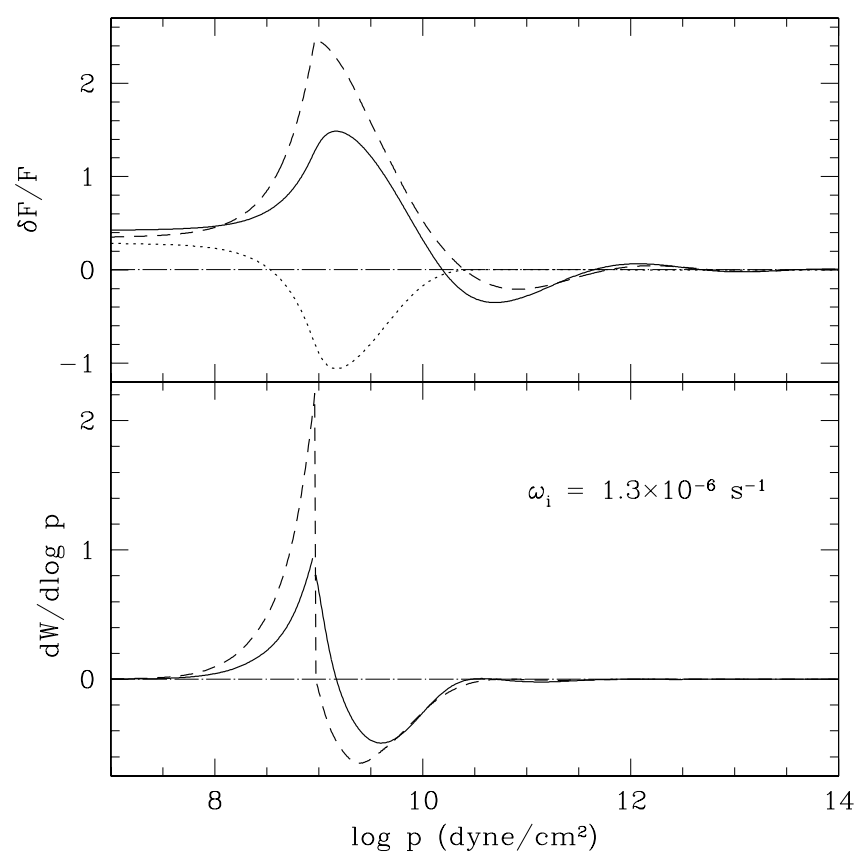

Fig. 3. - Similar to Fig. 2, but for a mode with $l=1$ and $P=1500 \mathrm{~s}$. Since $\omega \tau_{\text {th }}=0.8$ at $z_{b}$, this mode is moderately nonadiabatic at the top of the radiative interior. The mode remains overstable when radiative diffusion is taken into account. However, the flux perturbation at the photosphere is modified in both amplitude and phase.

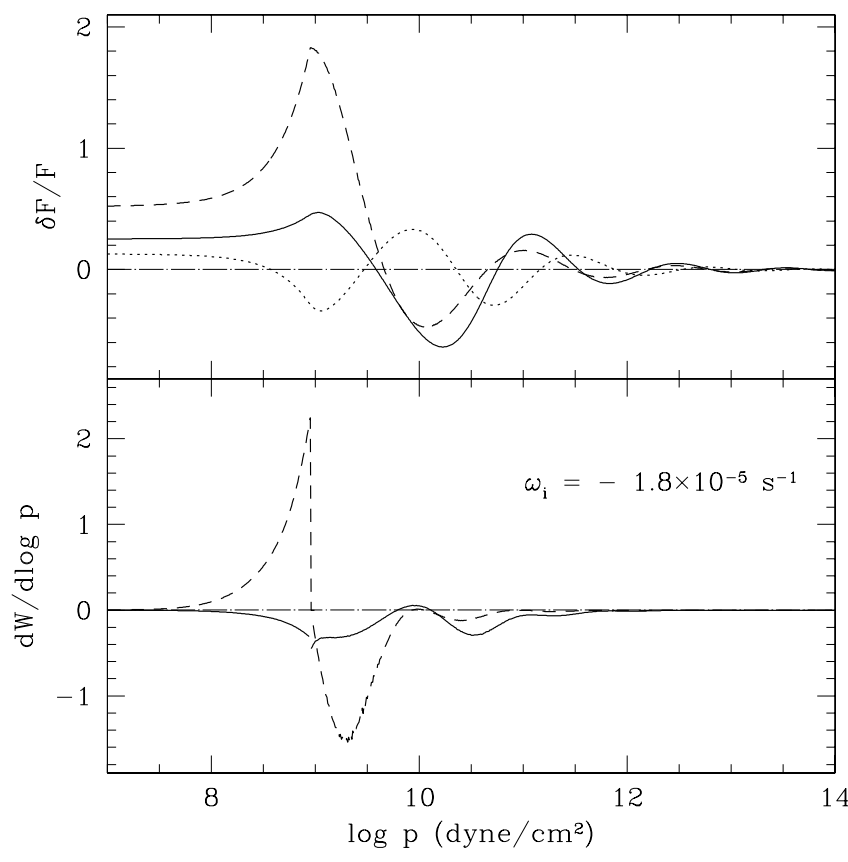

Fig. 4.- Similar to Fig. 2, but for a mode with $l=1$ and $P=2400 \mathrm{~s}$ corresponding to $\omega \tau_{\mathrm{th}}=0.5$ at $z_{b}$. The nonadiabatic region extends down into the propagating cavity. There is a large phase difference between $\delta F / F$ and $\delta p / p$ at $z_{b}$ that leads to convective damping. As a consequence, this mode is damped, although a quasi-adiabatic calculation predicts overstability.

The recent detection of velocity signals associated with $g$-modes by van Kerkwijk, Clemens, \& Wu (1999) is an important advance in the study of ZZ Cetis. What is being detected are horizontal velocities near the stellar limb. Phase differences and amplitude ratios of flux and velocity variations due to individual modes offer unique clues about both the star and the modes. Nonadiabatic calculations, such as that depicted in Figure 5, provide a theoretical basis for interpreting these observable quantities. Deep inside the radiative interior, the maximum flux perturbation precedes the maximum horizontal velocity by a quarter of a period, and the flux to velocity amplitude ratio is determined by the quasi-adiabatic approximation. These relations are modified in the upper envelope by the convective transport of heat and momentum and by radiative diffusion. Convection delays and diminishes the flux variation (see Paper I). It also flattens the horizontal velocity profile above $z_{b}$ and forces it to jump at $z_{b}$ (see Paper III). Radiative diffusion smears flux perturbations below the convection zone. In the example illustrated in Figure 5, a photospheric flux variation of 20 $\mathrm{mma}^{9}$ corresponds to a horizontal velocity of $v_{h}=2.3 \mathrm{~km}$ $\mathrm{s}^{-1}$. Nonadiabaticity, mostly in the convection zone, reduces the phase lag between velocity maximum and light maximum from the quasi-adiabatic value of $90^{\circ}$ to a value of $55^{\circ}$ at the photosphere. Employing the conventions in van Kerkwijk et al. (1999), this mode would exhibit $R_{v}=$ $\left|v_{h}\right| /(\omega|\delta F / F|)=16$, and $\Delta \Phi_{v}=\Phi(\delta F / F)-\Phi\left(v_{h}\right)=-55^{\circ}$. Effects of limb-darkening, disk-averaging, and bolometric correction introducing a factor close to unity: these numbers are consistent with those of the $818 \mathrm{~s}$ mode, which they observed to have $R_{v}=11 \pm 4$ and $\Delta \Phi_{v}=-44^{\circ} \pm 19^{\circ}$.

\footnotetext{
${ }^{9}$ Millimagnitude-amplitude is the commonly adopted unit for measuring flux perturbations. A $1 \mathrm{mma}$ variation means $\delta F / F=10^{-3}$.
} 

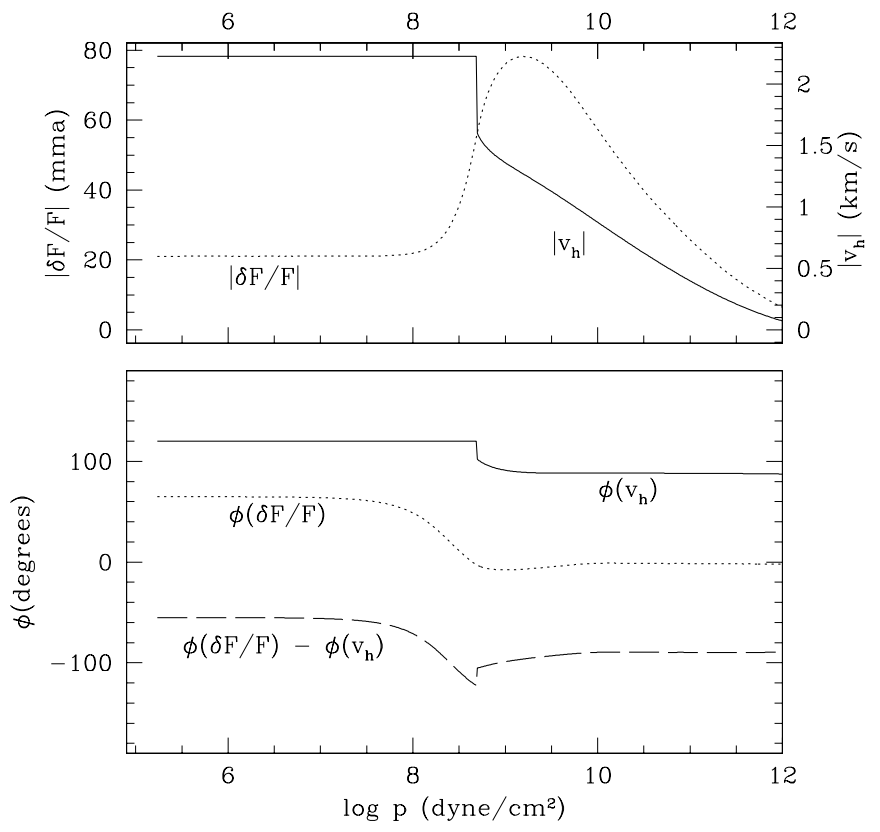

FIG. 5.-Amplitudes and phases of $\delta F / F$ and $v_{h}$ as functions of $\log p$. The displayed mode has $l=1$ and $P=800 \mathrm{~s}$ corresponding to $\omega \tau_{\mathrm{th}}=0.8$ at $z=z_{b}$ in a stellar model with $T_{\text {eff }}=12,160 \mathrm{~K}, g=10^{8} \mathrm{~cm} \mathrm{~s}^{-2}$, and $f=0.32$. The amplitude, $|X|$, and phase, $\phi$, of a perturbation $X$ are defined as $X=|X| \cos (\omega t-\phi)$. In the adiabatic interior, the flux perturbation leads the velocity perturbation by $90^{\circ}$.

\subsection{Nonadiabatic Effects on Driving and Damping Rates}

Where applicable, direct calculations of $\omega_{i}$ yield results consistent with those based on the work integral. This provides a measure of confidence in both. Modest discrepancies are found for some marginally overstable modes. The eigenvalue calculations are more reliable, because work integrals suffer from inaccuracies due to cancellation between comparable magnitudes of driving and damping. Values of $\omega_{i}$ for individual $g$-modes evolve with decreasing $T_{\text {eff }}$. Effects of nonadiabaticity on driving and damping rates of $g$-modes are presented in Figures 6-7. General trends are described below.

Upper lids of cavities of short period modes (e.g., the 430 $\mathrm{s}$ mode) lie far beneath the bottom of the convection zone in even in the coolest variables. Their driving/damping rates are largely immune to both nonadiabatic effects and the depth of the convection zone and hardly vary across the instability strip. The quasi-adiabatic estimate, $\omega_{i} \sim 1 /\left(n \tau_{\omega}\right)$, pertains to these modes (see Paper I).

Longer period modes (e.g., the $1000 \mathrm{~s}$ mode) become overstable at lower $T_{\text {eff }}$. Values of their $\left|\omega_{i}\right|$ exhibit a steady decline with decreasing $T_{\text {eff }}$. This is a consequence of the increase in mode inertia with decreasing $T_{\text {eff }}$. As the convection zone deepens, it depresses the upper lid of a mode's cavity. This decreases the relative size of the perturbation amplitude near the surface, where nonadiabaticity is greatest, with respect to that in the interior, where most of the mode energy is stored.

Modes with even longer periods (e.g., the $2000 \mathrm{~s}$ mode) are weakly overstable in cooler and narrower temperature ranges. Nonadiabatic effects tend to stabilize these modes. In the limit of strong dissipation, $\omega_{i} / \omega_{r} \approx-\ln \mathscr{R}^{-1} /(2 \pi n)$, where $\mathscr{R}$ is the reflection coefficient at the cavity lid. We derive this relation using a toy model in the Appendix. Magnitudes of nonadiabatic damping rates are an impor-
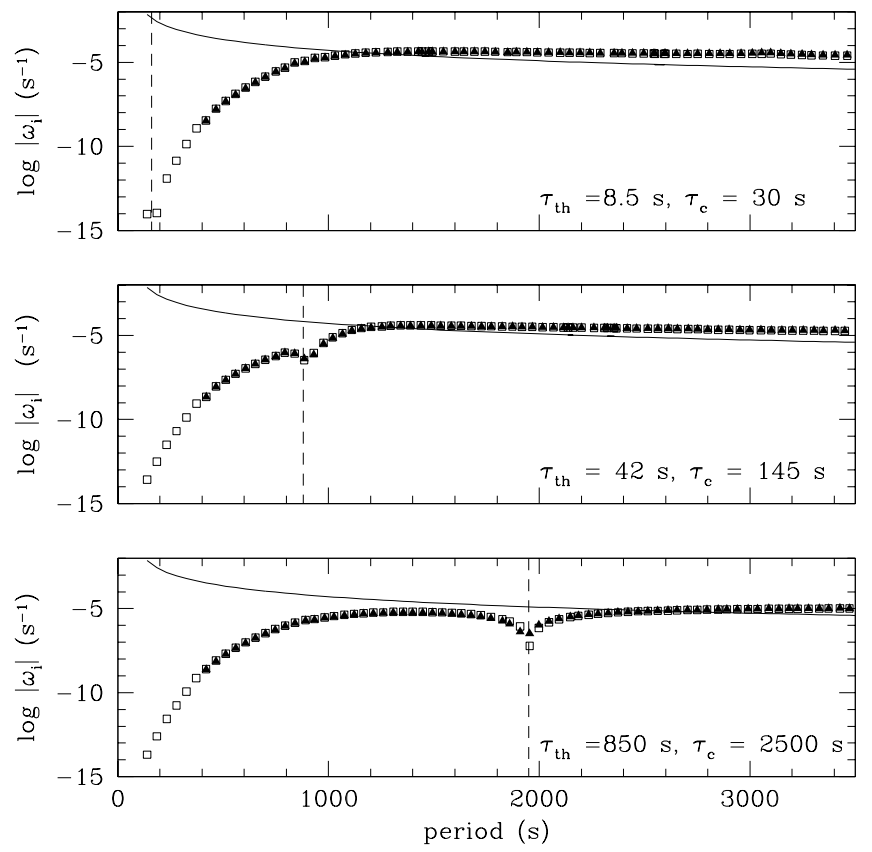

FIG. 6.-Driving and damping rates for $l=1$ modes plotted against mode periods. Top to bottom, the panels pertain to stellar models computed with $g=10^{8} \mathrm{~cm} \mathrm{~s}^{-2}$ and $f=0.32$ for $T_{\text {eff }}=12,600,12,300$, and $11,750 \mathrm{~K}$. Values of $\left|\omega_{i}\right|$ obtained from the nonadiabatic code are denoted by solid triangles. Those calculated from work integrals using nonadiabatic eigenfunctions are shown by open squares. For very short periods, $P<400 \mathrm{~s}$, our shallow envelopes force us to rely entirely on the work integral. The dashed vertical line marks the boundary between shorter period overstable modes and longer period damped ones. The solid line displays the analytic estimate, $\omega_{i}=-\omega_{r} \ln \mathscr{R}^{-1} /(2 \pi n)$, for the damping rate of modes that suffer strong dissipation. We set $\mathscr{R}=1 / e$ here, although in reality it decreases with increasing mode period and effective temperature.
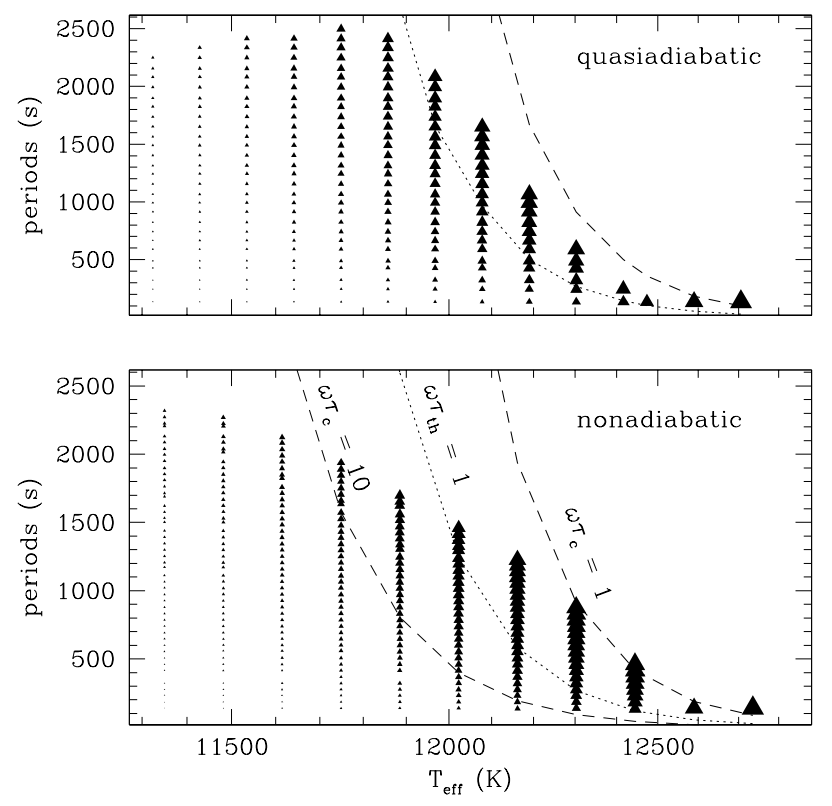

FIG. 7.-Panoply of overstable $l=1$ modes according to quasiadiabatic (upper panel) and fully nonadiabatic (lower panel) calculations in white dwarf models produced with $g=10^{8} \mathrm{~cm} \mathrm{~s}^{-2}$ and $f=0.32$ for a range of $T_{\text {eff }}$ that covers the ZZ Ceti instability strip. Each overstable mode is marked by a solid triangle whose size is proportional to the diminution factor of the flux perturbation in the convection zone, $\left[1+\left(\omega \tau_{c}\right)^{2}\right]^{-1 / 2}$ (see Paper I). Dashed lines denote the loci of constant values of $\omega \tau_{c}$. The dotted line corresponds to $\omega \tau_{\mathrm{th}}=1$ with $\tau_{\mathrm{th}}$ evaluated at $z=z_{b}$. 


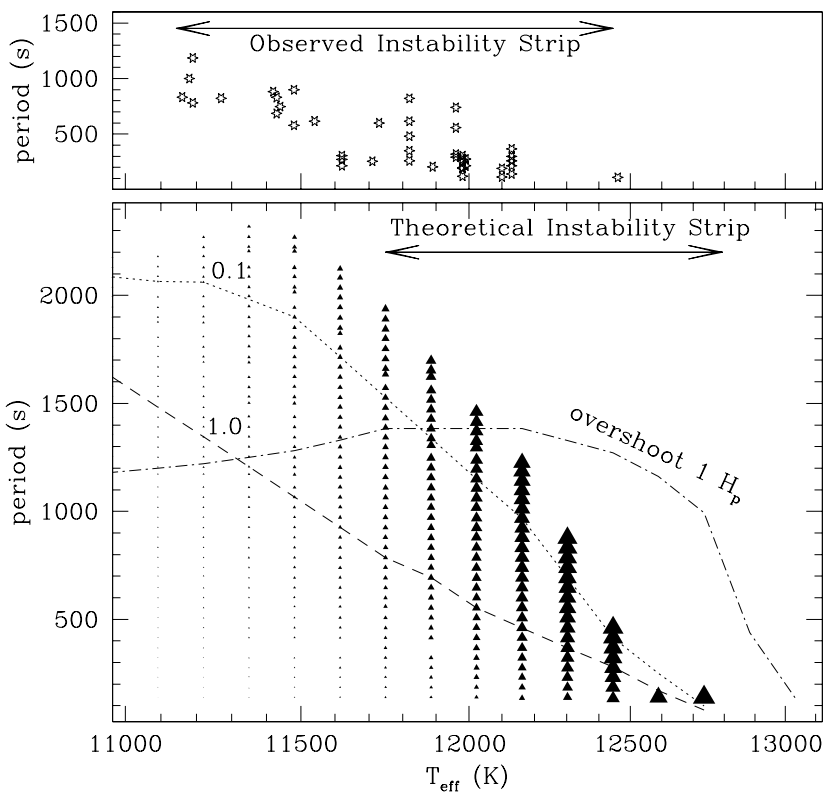

FIG. 8.-Observational and theoretical instability strips for DA white dwarfs. The upper panel displays each of the known ZZ Cetis according to the dominant period in its pulsation spectrum (Bradley 1995) and its inferred effective temperature (Bergeron et al. 1995). The lower panel repeats the material shown in the lower panel of Fig. 7. The dotted and dashed lines illustrate the maximum period for overstable modes under the stabilizing effect of turbulent damping in the region of convective overshoot for values of the parameter $\lambda / z_{b}$ equal to 0.1 and 1.0 , respectively. The dot-dashed line shows the envelope of overstable modes for models that incorporate 1 pressure scale height of convective overshoot. When comparing observational and theoretical instability strips, one should bear in mind that both depend on the assumed mixing-length.

tant input to calculations of parametric instability, an amplitude limiting mechanism for overstable modes that is explored in paper IV of this series.

\section{MAXIMUM PERIODS OF OVERSTABLE MODES}

6.1. Limitations of the Overstability Criterion $\omega \tau_{c}>1$

The derivation of the overstability criterion $\omega \tau_{c}>1$ depends on the quasi-adiabatic approximation and the condition that $z_{\omega} \gg z_{b}$ (Brickhill 1991; Paper I). Since $\tau_{c} \approx 4 \tau_{\text {th }}$ at $z_{b}$, the quasi-adiabatic approximation is suspect for modes with $\omega \tau_{c} \approx 1$. The upper and lower panels of Figure 7 display the boundary between overstable and damped modes as determined by quasi-adiabatic and nonadiabatic calculations. Both show that the boundary value of $\omega \tau_{c}$ is close to unity for hot ZZ Cetis but rises as stars cool. Inaccuracy of the quasi-adiabatic approximation does not dramatically modify the overstability criterion, but the violation of the condition $z_{\omega} \gg z_{b}$ does. The latter occurs because $z_{b}$ increases with decreasing $T_{\text {eff }}$, and $z_{\omega}$ decreases with decreasing $\omega$. Modes with $z_{\omega} \lesssim z_{b}$ propagate just below $z_{b}$. Their short Wentzel-Kramers-Brillouin (WKB) wavelengths enhance radiative damping above the estimate given by equation (54) in Paper I, on which the derivation of the overstability criterion $\omega \tau_{c}>1$ rests.

\subsection{Comparison with Observations}

Both our quasi-adiabatic and nonadiabatic calculations consistently predict maximum periods for overstable modes that are about a factor of 2 longer than those observed (see Fig. 8). We have not been able to resolve this discrepancy.
Several possible explanations that we have considered are described in what follows.

\subsubsection{Effects of Turbulent Damping}

Both Brickhill (1990) and Paper III stress that linear damping due to turbulent viscosity, although negligible inside the convection zone for all modes, may be significant in the region of convective overshoot for long-period modes. We incorporate this effect into our analysis, while recognizing that it cannot be quantified precisely.

The rate of turbulent damping in the overshoot region is expressed as

$$
\gamma_{\mathrm{vis}-\mathrm{os}} \approx \frac{-\pi R^{2} \rho_{b} \omega^{3} \lambda\left|\Delta \xi_{h}\right|^{2}}{4}
$$

(eq. [36] in Paper III), where $\left|\Delta \xi_{h}\right|$ is the normalized jump in the horizontal displacement across the convective overshoot region within which the turbulent viscosity decays on length scale $\lambda$. To cover our ignorance, we present results for $\lambda / z_{b}$ of 0.1 and 1.0. Figure 8 demonstrates that with the larger value, the longest period overstable mode at a given $T_{\text {eff }}$ is compatible with the observed upper limit of $P=1200$ s. However, it seems unlikely that $\lambda / z_{b} \approx 1$. The overshoot region probably extends less than 1 pressure scale height below $z_{b}$, while $H_{p} \approx 0.4 z_{b}$, and $\lambda$ must be several times smaller than $H_{p}$.

\subsubsection{Effects of Convective Overshoot}

We modify our envelope models to simulate convective overshoot by adding an isentropic layer below the base of the convection zone. Within this layer the radiative flux

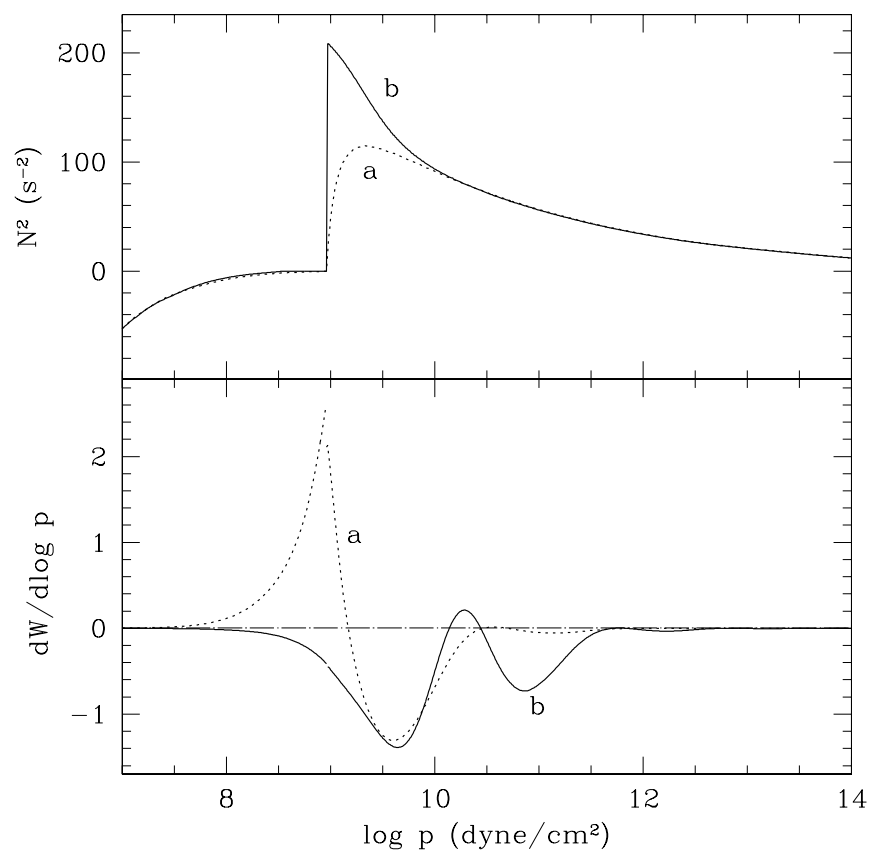

Fig. 9.-Effects of convective overshoot on $g$-mode structure and stability. We compare mode stability in two stellar models. Model $a$ has $T_{\text {eff }}=12,000 \mathrm{~K}$ and no convective overshoot, whereas model $b$ has $T_{\text {eff }}=$ $12,250 \mathrm{~K}$ and 1 pressure scale height of overshoot below its convection zone. These models are chosen in such a way that the bottom of the overshoot region in model $b$ is at the same depth as the base of the convection zone in model $a$. The upper panel displays their respective Brunt-Väisälä frequencies. The lower panel shows the differential work in arbitrary units for an $l=1$ mode with a period of $1500 \mathrm{~s}$ in both models $b$ and $a$. Convective overshoot in model $b$ is seen to strongly stabilize the mode, which is slightly overstable in model $a$. 
exceeds the total flux and turbulence transports energy downward. The top of the radiative interior immediately below the overshoot layer differs from that below a convective layer of similar depth. The temperature gradient is shallower and the Brunt-Väisälä frequency has a finite positive value. These differences enhance the effects of radiative diffusion and modify the relations between horizontal velocity and light perturbations at the photosphere.

Figure 9 provides such an example. Our nonadiabatic calculations indicate that 1 pressure scale height of overshoot suffices to stabilize all modes with $P>1400$ s (see Fig. 8). Moreover, by effectively deepening the convection zone, it shifts the instability strip to higher $T_{\text {eff }}$.

\subsubsection{Sensitivity to Surface Gravity}

Our nonadiabatic calculations indicate that, at fixed $T_{\text {eff }}$, the longest period for an overstable mode scales approximately as $g^{-1 / 3}$. Thus maximum periods of overstable modes around $1200 \mathrm{~s}$ would require $g \approx 5.0 \times 10^{8} \mathrm{~cm} \mathrm{~s}^{-2}$, which is well outside observational constraints. ${ }^{10}$

\subsubsection{Other Observational and Theoretical Considerations}

Hansen, Winget, \& Kawaler (1985) show that damping due to upward propagation of a gravity wave above the photosphere is unimportant for modes having frequencies exhibited by ZZ Cetis. We concur with this conclusion.

Detection of lower frequency modes requires longer observational data streams. Noise due to variations of atmospheric transparency increases at lower frequencies (see Fig. 4 of Winget 1991). These may result in an observational bias against the detection of low-frequency modes.

Evidence that photometric amplitudes of modes decline for $P \gtrsim 1000 \mathrm{~s}$ suggests that the observational cutoff at $P \approx 1200 \mathrm{~s}$ is genuine (see Fig. 5 of Clemens 1995). Taken literally, it also hints that the cutoff is the result of a nonlinear mechanism that saturates pulsation amplitudes.

\section{SUMMARY}

There is little doubt that convective driving, as originally proposed by Brickhill (1991), is responsible for the linear overstability of $g$-modes in DA white dwarfs. It is physically self-consistent and convincingly rationalizes observational facts. It accounts for the general location of the instability strip, although a precise specification depends upon the modeling of convection (e.g., the mixing-length parameter). Convective driving also explains why longer period modes become overstable as a star cools (see Fig. 8).

Although we agree with Brickhill that $\omega \tau_{c}>1$ is a necessary condition for mode overstability, we find that it is not a

\footnotetext{
${ }^{10}$ Applying a theoretical mass-radius relation (Hamada \& Salpeter 1961) for white dwarfs, this corresponds to a stellar mass about $1.2 M_{\odot}$.
}

sufficient condition for modes whose periods exceed $1000 \mathrm{~s}$. This stems from enhanced radiative damping of modes whose upper cavity lids approach $z_{b}$, as is apparent from both quasi-adiabatic and nonadiabatic calculations (see Fig. 7). Our nonadiabatic calculations yield a maximum period of about $2300 \mathrm{~s}$ for overstable modes. This clashes with the maximum period of $1200 \mathrm{~s}$ for observationally detected modes.

We also agree with Brickhill's (1990) deduction that turbulent convection forces the horizontal velocity to be nearly independent of depth within the convective envelope. Consequently, mode damping due to turbulent dissipation within the convection zone is reduced to a negligible level. However, suppression of the horizontal shear in the convective envelope results in a shear layer at the top of the radiative interior. When convective overshoot is accounted for, this provides linear turbulent damping (see Paper III). Figure 8 suggests that turbulent dissipation might depress the maximum period of observable modes to some degree. Convective overshoot also alters the thermal structure of the upper radiative layer. A rough treatment of this effect with 1 scale height of overshoot predicts a maximum period of $1400 \mathrm{~s}$ for overstable modes. Nonlinear interactions that limit the amplitudes of overstable modes may also play a part in determining the maximum period.

In conclusion, the blue edge of the theoretical instability strip is set by the condition that $\omega \tau_{c} \approx 1$ for the lowest order $l=1$ mode. ${ }^{11}$ The lowest order $l=2$ mode is excited at a slightly higher temperature. The location of the red edge is more nebulous and may result from a combination of decreased photometric visibility, convective overshoot, and nonlinear effects. Additional detections of velocity variations associated with $g$-modes could provide important clues. Convective driving makes the testable prediction that velocity variations become relatively more observable than photometric variations toward the red edge of the instability strip.

We are indebted to P. Bradley for supplying us with models of DA white dwarfs. The final version of this paper benefited from constructive comments offered by W. Dziembowski. Financial support for this research was provided by NSF grant 94-14232.

\footnotetext{
11 The influence of stellar mass and convective efficiency on the location of the blue edge can be assessed by applying this criterion.
}

\section{APPENDIX}

\section{A TOY MODEL FOR NONADIABATIC MODES}

We describe a simple toy model for nonadiabatic modes. It is particularly useful for interpreting damping rates in the limit of strong dissipation.

Consider waves that satisfy the one-dimensional, homogeneous, acoustic wave equation,

$$
\frac{\partial^{2} \xi}{\partial t^{2}}=u^{2} \frac{\partial^{2} \xi}{\partial z^{2}}
$$


in the interval $0 \leq z \leq L$. Here $\xi$ is the Lagrangian displacement, and $u$ is the constant propagation speed. The dispersion relation connecting frequency, $\omega$, and wave vector, $k$, reads $\omega^{2}=k^{2} u^{2}$. We take the lower boundary to be a rigid, perfectly reflecting wall, so

$$
\xi=0 \quad \text { at } \quad z=L .
$$

Dissipation is introduced by means of a partially reflective upper boundary, where $\mathscr{R}$ denotes the reflection coefficient of the incident wave. This is expressed through the boundary condition

$$
\left(\frac{\partial}{\partial t}-u \frac{\partial}{\partial z}\right) \xi=-\mathscr{R}\left(\frac{\partial}{\partial t}+u \frac{\partial}{\partial z}\right) \xi \quad \text { at } \quad z=0
$$

Note that in the limit $\mathscr{R} \rightarrow 1$, the upper boundary becomes a perfectly reflecting wall.

Eigensolutions of equation (A1) are composed of oppositely directed waves:

$$
\xi=A e^{-i \omega t-i k z}+B e^{-i \omega t+i k z} .
$$

Application of the boundary conditions given by equations (A2) and (A3) yields

$$
B=-\mathscr{R} A \text {, }
$$

and

$$
k L=n \pi-\frac{i}{2} \ln \mathscr{R}^{-1},
$$

where $n$ is the number of half-wavelengths between the walls. Then the dispersion relation implies

$$
\omega_{i}=\frac{k_{i}}{k_{r}} \omega_{r}=-\frac{\omega_{r}}{2 \pi n} \ln \mathscr{R}^{-1},
$$

where $n$ is an integer.

Equation (A7) is the key result of our toy model. It demonstrates that $\omega_{i}$ grows logarithmically with $\mathscr{R}^{-1}$ and provides an order-of-magnitude estimate for the damping rate of strongly nonadiabatic stellar modes. Radiative diffusion substantially suppresses the effective reflection coefficients of modes having $\omega \tau_{\omega} \leq 1$. In hot DA stars, this applies to modes with periods in excess of $1000 \mathrm{~s}$ (see Fig. 6). Finally, equation (A7) justifies the procedure we apply in $\S 4.1$ to translate $\omega_{i}^{\prime}$, the damping rate for a mode of an envelope model, to $\omega_{i}$, the damping rate for a mode of a complete stellar model.

\section{REFERENCES}

Bergeron, P., Wesemael, F., Lamontagne, R., Fontaine, G., Saffer, R. A., \& Allard, N. F. 1995, ApJ, 449, 258

Bradley, P. A. 1995, Baltic Astron., 4, 536

. 1996, ApJ, 468, 350

Brickhill, A. J. 1990, MNRAS, 246, 510 .1991, MNRAS, 251, 673

Clemens, J. C. 1995, Baltic Astron., 4, 142

Dziembowski, W. 1977, Acta Astron., 27, 95

Gautschy, A., Ludwig, H. G., \& Freytag, B. 1996, A\&A, 311, 493

Goldreich, P., \& Wu, Y. 1999a, ApJ, 511, 904 (Paper I) 1999b, ApJ, submitted (astro-ph/9810038; Paper III)
Hamada, T., \& Salpeter, E. E. 1961, ApJ, 134, 683

Hansen, C. J., Winget, D. E., \& Kawaler, S. D. 1985, ApJ, 297, 544

Iglesias, C. A., \& Rogers, F. J. 1996, ApJ, 464, 943

Press, W. H., Teukolsky, S. A., Vetterling, W. T., \& Flannery, B. P. 1992, Numerical Recipes in FORTRAN (Cambridge: Cambridge Univ. Press) Rogers, F. J., Swenson, F. J., \& Iglesias, C. A. 1996, ApJ, 456, 902

Unno, W., Osaki, Y., \& Ando, H. 1989, Nonradial Oscillations of Stars (Tokyo: Univ. Tokyo Press), 238

van Kerkwijk, M. H., Clemens, J. C., \& Wu, Y. 1999, MNRAS, submitted Winget, D. E., et al. 1991, ApJ, 378, 326 